\title{
СПОНДИЛОДИСЦИТЫ: СОВРЕМЕННЫЕ ПОДХОДЫ К ДИАГНОСТИКЕ И ЛЕЧЕНИЮ
}

\author{
КУБРАКОВ К.М., МИГУНОВА В.А.
}

Витебский государственный ордена Дружбы народов медицинский университет, г. Витебск, Республика Беларусь

Вестник ВГМУ. - 2018. - Том 17, №1. - С. 14-22.

\section{SPONDYLODISCITIS: MODERN APPROACHES TO DIAGNOSING AND TREATMENT}

\author{
KUBRAKOV K.M., MIGUNOVA V.A. \\ Vitebsk State Order of Peoples' Friendship Medical University, Vitebsk, Republic of Belarus \\ Vestnik VGMU. 2018;17(1):14-22.
}

\begin{abstract}
Резюме.
Спондилодисцит (СД) - инфекционное заболевание позвонков и межпозвонковых дисков. Частота его возросла в последние годы в связи с учащением инвазивных манипуляций на позвоночнике. Актуальность данной проблемы определяется тем, что при таком заболевании нарушаются две основные функции позвоночника - обеспечение стабильного вертикального положения туловища и защита спинальных нервных структур. При этом диагностика спондилодисцита затруднительна и зачастую происходит со значительной задержкой после появления первых симптомов, что связано с редкой встречаемостью данного заболевания, неспецифичностью его проявлений, а в связи с этим недостаточной информированностью врачей по вопросам инфекционных поражений позвоночника. Существующая проблема с определением этиологии заболевания ведет к нерациональной антибиотикотерапии, длительности лечения и повышению затрат. Хирургическое лечение спондилодисцита выполняется с задержкой вследствие отсутствия строгих показаний.
\end{abstract}

Ключевые слова: спондилодисцит, инфекиия, диагностика, биопсия, лечение, операция.

\begin{abstract}
.
Spondylodiscitis is an infectious disease of vertebrae and intervertebral discs. Its incidence has increased recently due to the growth of invasive manipulations on the spinal cord. The urgency of this problem is determined by the fact that during such disease two basic functions of the spine are damaged - ensuring of the stable vertical position of the body and protecting of the spinal nervous structures. In this case, the diagnosis of spondylodiscitis is difficult and it often occurs with a significant delay only after the appearance of the first symptoms. It is connected with the rare occurrence of this disease, lack of specificity of its manifestations, and as a result insufficient possession of information on the issues concerning the spinal infections by the medical staff. The existing problem with the identification of the etiology of this disease leads to irrational antibiotic therapy, the duration of treatment and rising costs. Surgical treatment for spondylodiscitis is carried out with delay because of the absence of strict indications.
\end{abstract}

Key words: spondylodiscitis, infection, diagnosing, biopsy, treatment, operation.

Спондилодисцит (СД) - воспалительный процесс позвоночника, который вовлекает межпозвоночные диски, смежные с ними позвонки и суставы. Заболевание протекает на фоне дегенеративно-дистрофического процесса позвоночного столба или формируется после оперативных вмешательств на позвоночнике [1, 2, 3]. В международной классификации болезней (МКБ 10) патология относится к классу 13 - болезни костно-мышечной системы и соединительной ткани, блоку спондилопатии (M45-M49), рубрике другие воспалительные спондилопатии (M46). 
Актуальность проблемы СД определяется нарушением основных функций позвоночника: обеспечения стабильного вертикального положения туловища и защиты спинальных невральных структур [4]. Неспецифичность клинической картины, редкая встречаемость и, в связи с этим, недостаточная информированность врачей по вопросам инфекционных заболеваний позвоночника, а также отсутствие единого клинического руководства и протоколов лечения СД обусловливают частые ошибки в диагностике данной патологии. Вследствие этого задержка в постановке диагноза достигает иногда 12 месяцев, в среднем составляя 4,3 месяца [3, 5-7].

В обзоре за последнее десятилетие мы представили анализ проблем, связанных с ранней диагностикой, определением тактики ведения и лечения пациентов с СД.

\section{Эпидемиология. Этиопатогенез}

\section{Распространенность СД}

Заболеваемость СД в развитых странах колеблется в пределах 4-25 случая на 1 млн. населения в год $[1,3,5,8]$. Распределение по возрасту во многих исследованиях выявляет два подъема заболеваемости: до 20 лет и в возрасте 50-70 лет. При этом заболевание может возникать в любом возрасте. Мужчины болеют почти в 2 раза чаще женщин. Заболеванию наиболее подвержены пожилые люди, пациенты с хронической эндокринной патологией (в особенности с сахарным диабетом), пациенты с иммунодефицитом, длительно принимающие цитостатическую и гормональную терапию, внутривенные наркоманы, а также пациенты, перенесшие хирургическое вмешательство на позвоночнике. Несмотря на широкое использование антибактериальной терапии, летальность при СД остается достаточно высокой и составляет от 2 до $17 \%$ [1, 3, 4, 7, 9-11].

\section{Классификация воспалительных пора- жений позвоночника}

Существуют различные классификации инфекционных заболеваний позвоночника. По источнику инфекции выделяют первичные (возникающие при отсутствии других видимых очагов инфекции) и вторичные (развивающиеся вследствие заноса инфекции из других очагов или в результате травмы) поражения. Отдельно выделяют ятрогенные остеомиелиты позвоночника, обусловленные проведением инвазивных диагностических или лечебных манипуляций [4].

По клиническому течению выделяют острые, подострые и хронические СД $[4,14,16]$. Классически острый процесс сохраняется в течение трех недель, подострый - от трех недель до трех месяцев, а хронический - свыше трех месяцев.

По локализации можно выделить СД шейного, грудного, поясничного и крестцового отделов позвоночника. При этом инфекции грудного и поясничного отделов встречаются в $35-50 \%$ случаев, а шейный отдел поражается только в $3-10 \%$ случаев $[1,4,11,12]$.

\section{Возбудители СД}

Этиологически выделяют специфические и неспецифические инфекционные поражения позвоночника, которые делятся на гнойные, гранулематозные (туберкулезные, бруцеллезные, грибковые) и паразитарные поражения $[1,4,12]$.

На сегодняшний день наиболее часто встречаются гнойные СД, вызываемые грам-положительной флорой, в особенности Staphylococcus aureus. Их частота составляет, по данным R. Sobbotke et al. (2008), R.M. Duarte, A.R. Vaccaro (2013), от 30\% до 80\% [3, 15]. Грам-отрицательные микроорганизмы, такие как Escherichia coli, вызывают до 25\% инфекций позвоночника. Анаэробные бактерии также могут явиться причиной СД, особенно в случае проникающей травмы позвоночника [1, 4, 15]. СД ассоциированные с Mycobacterium tuberculosis особенно распространены среди ВИЧ-инфицированных пациентов, у которых частота их достигает $60 \%[3,15]$. В остальной популяции частота туберкулезных СД значительно снизилась за последние 50 лет. Однако следует обратить внимание, что существуют эндемичные районы, такие как Восточная Европа и Средиземноморье, где частота СД туберкулезной и бруцеллезной этиологии остается высокой [1, 15]. Другими возможными возбудителями СД являются коагулазонегативные стафилококки, стрептококки, клебсиелла, энтерококки, сальмонелла, синегнойная палочка, протей, бактероиды, ацинетобактер, кингеллы, грибы рода Candida, Aspergillus [1, 4, 8, 9, 13]. Чаще всего инфекция вызвана одним видом возбудителя. При этом в одной трети случаев заболевания возбудителя определить не удается [3, 14].

Патоген может проникать в позвоночник тремя способами: гематогенным, путем прямого заноса из внешней среды (в случае открытого 
перелома, раны, язвы) и при распространении из соседних гнойных очагов типа фурункула $[9,15]$.

\section{Патогенез СД}

$\mathrm{У}$ детей хорошо развиты сосудистые внутрикостные анастомозы, при этом некоторые из них проникают в межпозвонковые диски. Вследствие этого при заносе инфекционного агента гематогенным путем не происходит некроз кости, а инфекция локализуется преимущественно в пределах межпозвонкового диска [15]. У взрослых внутрикостные анастомозы подвергаются инволюции, а межпозвонковый диск становится аваскулярным. При попадании патогенного микроорганизма происходит обширный некроз кости и инфекция распространяется на прилежащие структуры, что обусловливает классическую картину СД: эрозия запирательных пластинок тел позвонков, остеолиз и компрессионные переломы, что может привести к нестабильности, деформации позвоночника и риску компрессии спинного мозга и корешков [15]. Инфекция может поражать окружающие ткани, что приводит к образованию паравертебральных и поясничных абсцессов. Проникновение возбудителя в позвоночный канал обусловливает развитие эпидуральных, субдуральных абсцессов, менингита и миелита [15].

Гематогенным путем инфекция может проникать из мочеполового тракта $(17 \%)$, кожи и мягких тканей (11\%), внутрисосудистых имплантатов (5\%), желудочно-кишечного тракта (5\%), дыхательных путей $(2 \%)$ и ротовой полости $(2 \%)$. В $12 \%$ случаев обнаруживается инфекционный эндокардит [1].

Гнойный СД чаще возникает в поясничном отделе позвоночника, реже в грудном и еще реже - в шейном отделе. Туберкулезное поражение преимущественно затрагивает грудной отдел позвоночника и часто вовлекает в патологический процесс более двух сегментов, что отличает его от гнойного СД [15].

В современной медицине все чаще встречается ятрогенный путь проникновения инфекции, что связано постоянным ростом частоты хирургических вмешательств, а также широким распространением различных диагностических процедур на позвоночнике $[3,4,9,16]$.

Самым редким вариантом является проникновение микробов из соседних тканей. Это может быть обусловлено наличием грыжи пищеводного отверстия диафрагмы, заглоточного аб- сцесса и другими воспалительными причинами [15].

\section{Диагностика СД}

\section{Клиническая картина}

Клинические симптомы СД не специфичны. Начало заболевания, как правило, проходит незамеченным, и сигналом тревоги становится постоянная боль в спине, которая усиливается в ночное время, при ходьбе и нарастающие признаки общей интоксикации. Длиться такое состояние может по-разному: от 10 дней и до одного месяца, в зависимости от тяжести процесса $[1,4]$.

Самый распространенный симптом СД боль в спине, однако до $15 \%$ пациентов могут не испытывать болевых ощущений $[1,15]$. Болевой синдром зависит от локализации и распространенности патологического процесса в позвоночнике. Самая частая локализация болей и, соответственно, поражения - в поясничном отделе, реже - в грудном и очень редко - в шейном. Боли в грудном отделе позвоночника могут напоминать пневмонию или плеврит, в нижнегрудном и поясничном отделах имитируют острый живот, динамическую непроходимость, паранефрит, остеохондроз. Это приводит к ошибкам в диагнозе или даже к ненужной операции [4].

Лихорадка менее распространенный симптом, который наблюдается примерно у половины пациентов [4]. Реже всего лихорадка встречается при СД туберкулезной этиологии [1].

Распространение инфекционного процесса на позвоночный канал сопровождается развитием радикулярного синдрома с ограничением двигательной активности, признаками раздражения или угасания рефлексов, нарушениями чувствительности в зонах пораженных корешков $[1,10,15]$.

При прогрессировании заболевания появляются признаки компрессии спинного мозга с частотой от 10 до 64\%: развитие парезов конечностей с нарастанием неврологического дефицита до плегии, нарушение функции тазовых органов с задержкой мочеиспускания и дефекации.

Деформация позвоночника в виде кифоза чаще встречается при туберкулезном поражении. СД шейного отдела может проявляться дисфагией или кривошеей [1]. Резкая болезненность при пальпации остистых отростков позвонков и паравертебральной области на уровне поражения - самый частый признак, выявляемый при обследовании пациента (78-97\% случаев), который не- 
редко ассоциирован с ограничением амплитуды движений и спазмом паравертебральных мышц $[1,4,17]$.

Отсутствие характерных симптомов заболевания приводит к поздней диагностике и ухудшению результатов лечения. В среднем период между началом заболевания и постановкой диагноза составляет от 8 недель до 3 месяцев [1, 4, $10,17]$.

\section{Лабораторная диагностика}

Чувствительным, но малоспецифичным маркером инфекции является скорость оседания эритроцитов (СОЭ). Во многих исследованиях данный показатель повышен более чем в 90\% случаев, его значение в среднем составляет от 43 до 87 мм/ч. Падение СОЭ на 25\% и больше считается хорошим прогностическим признаком терапии [1].

С-реактивный белок повышен у более, чем 90\% пациентов со СД. Быстрое снижение уровня С-реактивный белка указывает на правильный выбор терапии и позволяет заменить инъекционные формы антибиотиков пероральными [15].

Лейкоцитоз выявляется только у 30-50\% пациентов и является наименее чувствительным воспалительным маркером заболевания $[15,17]$.

\section{Микробиологическая диагностика}

Наиболее важно при диагностике СД определить этиологический фактор, вызвавший инфекционный процесс. Это связано с достаточно широким кругом возможных возбудителей и ростом антибиотикорезистентности [4, 5, 17]. Эмпирически назначенная антибиотикотерапия часто приводит к развитию осложнений, расширению показаний для оперативного лечения, а также к удлинению сроков пребывания пациента в стационаре и повышению затрат на лечение $[1,4]$.

Самым простым методом микробиологического исследования является гемокультура. Однако данный метод имеет диагностическое значение лишь в 25-33\% случаев и бывает положителен лишь при инфекции, вызванной высоковирулентным штаммом $[4,5,8,11]$. При этом кровь на посев нужно брать на высоте лихорадки и до начала антибактериальной терапии, что редко представляется возможным. Рекомендуется производить трехкратные посевы с определением чувствительности к антибиотикам [3, 5, 11, 17, 18].

Раннее определение туберкулезного процесса в позвоночнике позволит выделить пациен- тов со СД туберкулезной этиологии, что снизит распространение инфекции, а также даст возможность для раннего начала противотуберкулезной терапии в специализированном стационаре. Для исключения СД, вызванного Mycobacterium tuberculosis, используют несколько методов [18]. Диаскинтест - проба, выявляющая инфицированность пациента туберкулезом. Проба Манту на сегодняшний день менее актуальна, так как выявляет только активный туберкулезный процесс, а также дает большую частоту ошибочных результатов. Данные методы являются наиболее простыми и дешевыми. Методы определения ДНК возбудителя: LPA (Хайн-тест), GenExpert. Данные методы являются наиболее быстрыми и точными при диагностике туберкулеза. Важное преимущество их - возможность одновременного определения лекарственной устойчивости микобактерий [19].

\section{Биопсия патологического очага}

Биопсия инфицированной зоны с последующим микробиологическим исследованием в различных исследованиях колеблется от $19 \%$ до $100 \%[1,20,21]$. Чаще всего данный метод используют у пациентов с отрицательным результатом посева крови. Биопсия у этих пациентов положительна в 43-78\% случаев $[1,4]$. Биопсийный материал должен быть посеян на среды, диагностические для аэробных, анаэробных микроорганизмов, грибов и микобактерий. Одновременно биопсийный материал должен быть подвергнут гистологическому исследованию. Основная его роль - разграничение гнойного и гранулематозного процесса [15].

Также биопсийный материал можно исследовать с помощью ПЦР. Это высокоэффективный метод, который позволяет выявить микроорганизмы в минимальном количестве, а также после начала антибиотикотерапии [17]. В настоящее время методы определения ДНК микроорганизмов развиваются быстрыми темпами. Однако главной проблемой их использования является высокая стоимость [1].

Существуют закрытый (чрескожный) и открытый способы получения биопсии. Для получения материала при инфекционных заболеваниях позвоночника самым оптимальным из закрытых методов является пункционная биопсия, разновидностями которой являются тонкоигольная аспирационная биопсия и трепан-биопсия. Пункционная биопсия проводится под ультразву- 
ковым, флюороскопическим, КТ или МРТ контролем $[4,16]$.

Ультразвуковой контроль малоэффективен при диагностике заболеваний позвоночника. Его возможности ограничиваются обнаружением поверхностных повреждений в шейном отделе позвоночника [16]. Биопсия под контролем флюороскопа является наиболее простым, недорогим, занимающим непродолжительное время методом, позволяющим наблюдать за процедурой в режиме реального времени. Недостатком данного метода является его неспособность изображать окружающие мягкие ткани [20]. Визуализация процедуры с помощью компьютерной томографии является стандартом во многих развитых странах. КТ-контроль позволяет запланировать траекторию движения иглы, избежать повреждения легких, а также проникнуть в труднодоступные очаги [15, 21-23]. Недостатками являются длительность процедуры и воздействие ионизирующей радиации. Преимущество КТ-контроля перед флюороскопией заключается в возможности визуализации мягких тканей наравне с костной, а также в более четкой визуализации иглы, особенно при небольших патологических очагах [15]. МРТ-контроль используется лишь в крупных специализированных нейрохирургических центрах. Его преимущества состоят в более сильном контрасте между мягкими и костными тканями, отсутствии ионизирующей радиации и способности к многоплановой визуализации [24].

Открытая биопсия выполняется во время оперативного вмешательства. Для позвоночника более актуальна инцизионная биопсия, когда в целях исследования удаляется участок патологического образования. Открытая биопсия является диагностической примерно в $80 \%$ случаев $[3,4,12]$.

\section{Инструментальная диагностика}

Плановая рентгенография может выявить такие ранние изменения, как субхондральный склероз, потеря четкости концевых пластинок тел позвонков и уменьшение высоты межпозвонкового диска $[1,15]$. Поздние изменения включают разрушение противоположной концевой пластинки, уменьшение высоты позвонка. Однако симптомы начинают развиваться лишь спустя 2-8 недель после возникновения симптомов, а ложноположительные результаты могут привести к развитию дегенеративных изменений в позвоночнике [1].

Для радионуклидной диагностики СД используют различные изотопы. Технеций- $99 \mathrm{~m}$ обладает высокой чувствительностью - до 90\%, но менее высокой специфичностью - 78\%. Галлий-67 используется в сочетании с рентгенографией, при этом чувствительность данного метода достигает $90 \%$, специфичность - $100 \%$, а точность $-94 \%$ [1].

КТ - один из лучших методов диагностики поражений костей. С помощью КТ можно обнаружить такие изменения, как раннее разрушение концевых пластинок (до того, как это станет видимо при рентгенографии), более поздние секвестры и патологическую кальцификацию, обнаруживаемую при туберкулезе. Изменения в межпозвонковом диске проявляются в виде гиподенсивных областей [15].

МРТ является методом выбора в инструментальной диагностике СД. Его чувствительность составляет примерно 96\%, специфичность - 93\%, а точность - 94\% [1]. Его преимущество перед другими методами заключается в превосходящей способности к визуализации эпидурального пространства и спинного мозга. Характерные изменения заключаются в снижении интенсивности сигнала от межпозвонкового диска и прилегающих тел позвонков в режиме T1, усилении сигнала в режиме Т2 и потеря четкости концевых пластинок в режиме T1 $[1,24]$. Контрастирование гадолинием повышает точность исследования (особенно при ранней диагностике, когда другие изменения могут отсутствовать), а также помогает дифференцировать инфекционное поражение от дегенеративных изменений и новообразований [1]. При туберкулезном поражении позвоночника обычно отсутствуют изменения в межпозвонковом диске, но могут обнаруживаться паравертебральные абсцессы, изменения задних структур позвонков, а также в процесс вовлекаются отдаленные позвонки и выявляется их деструкция [24].

При наличии гнойного СД изменения МРТ в процессе лечения часто выявляют отсутствие динамики или даже ухудшение процесса. Это приводит к ненужным хирургическим вмешательствам. Положительная динамика МРТкартины может отсутствовать первые 4-8 недель терапии. Соответственно не рекомендуется ориентироваться на данные МРТ для оценки эффективности лечения $[1,17]$.

\section{Лечение}

Целью лечения СД является уничтожение возбудителя, восстановление и сохранение струк- 
туры и функции позвоночника, нивелирование неврологического дефицита и облегчение боли $[1,9,14]$.

\section{Консервативная терапия}

Консервативное лечение показано при отсутствии на КТ и МРТ очагов деструкции и признаков сдавления спинного мозга, что является стандартом лечения $[1,14,16]$.

В отношении консервативных методов терапии разногласий нет, и в настоящее время она включает антибактериальную, иммунокорригирующую, дезинтоксикационную терапию, физиотерапию и иммобилизацию $[4,14,25,26]$. Иммобилизация должна использоваться при значительно выраженном болевом синдроме и когда имеется риск развития нестабильности позвоночника $[1,3]$.

\section{Антибактериальная терапия}

На ранних стадиях СД легко поддается антибиотикотерапии. Эмпирическую антибактериальную терапию рекомендуется начинать немедленно пациентам с нарушениями гемодинамики, сепсисом, септическим шоком, при наличии прогрессирующих или тяжелых неврологических нарушений [6]. Антибиотики назначают в максимальных терапевтических дозах внутривенно, в отдельных случаях предлагается внутриаортальное введение антибиотиков [6]. В остальных случаях назначение антибактериальной терапии может быть отложено до получения результатов биопсии, так как раннее начало эмпирического лечения приводит к искажению результатов биопсии $[1,14,16]$.

Эмпирическая терапия должна охватывать наиболее распространенных возбудителей СД и хорошо проникать в центральную нервную систему и кости $[1,8]$. По мнению C.E. Chenoweth [et al.] (2010), I. Moraru (2012), T. Gouliouris [et al.] (2012), K. Zarghooni [et al.] (2013), в большинстве случаев эффективными являются следующие комбинации антибиотиков для эмпирической терапии:

1. Предпочтительная схема: ванкомицин 15-20 мг/кг + цефтриаксон 2 г - внутривенно, каждые 12 ч.

2. Альтернативная схема при аллергии на пенициллины: ванкомицин 15-20 мг/кг + азтреонам 2 г - внутривенно, каждые 8 ч.

Препаратами для лечения СД в составе эмпирической терапии также являются: цефепим 6 г-в сутки внутривенно, меропенем 6 г - в сутки внутривенно $[1,5,6,14]$.
Если в результате бактериологических исследований был идентифицирован возбудитель, назначается рациональная антибиотикотерапия, которая проводится с учетом резистентности выделенного патогена к антибактериальным препаратам $[8,14]$.

Длительность парентеральной антибиотикотерапии составляет в среднем 2-4 недели и зависит от улучшения клинической картины, нормализации или значительного снижения СОЭ, количества лейкоцитов $[1,6]$. Основанием для перехода с парентерального введения антибиотиков на пероральное может служить уровень С-реактивного белка. Если данный показатель нормализуется в первые две недели лечения, рекомендуется переход с парентерального введения антибиотиков на пероральный прием [3]. Лечение следует продолжать в течение как минимум 6-8 недель после нормализации воспалительных маркеров [5].

По мнению многих авторов, общая длительность антибактериальной терапии должна составлять от 6 недель до 3 месяцев в случае гнойного СД и до 1 года при туберкулезной этиологии заболевания $[1-3,6,12]$.

\section{Хирургическое лечение}

Целями оперативного вмешательства являются ликвидация инфекционного очага, взятие биопсии для микробиологического и гистологического исследования, декомпрессия позвоночного канала со стабилизацией и восстановлением поврежденных спинальных структур $[3,5]$.

Большинство авторов (R. Sobottke [et al.] 2008, K. Zarghooni [et al.] 2012, S. Mann [et al.] 2004, R.M. Duarte, A.R. Vaccaro 2013) рекомендуют выполнять экстренное хирургическое вмешательство при прогрессировании неврологического дефицита, а также нестабильности и деформации позвоночника. Абсолютные и относительные показания к оперативному лечению СД представлены в таблице $1[3,5,10,15]$.

Однако до сих пор остаются дискутабельными вопросы с относительными показаниями к оперативному лечению при СД у пациентов с болевым синдромом и при отсутствии эффекта от консервативной терапии.

В сравнении с консервативной терапией хирургический способ лечения способствует более безопасному и быстрому снятию воспаления и болевого синдрома.

Безусловно, имплантация фиксационных 
Таблица 1 - Показания к хирургическому лечению

\begin{tabular}{|l|l|}
\hline \multicolumn{1}{|c|}{ Абсолютные показания к операции при СД } & \multicolumn{1}{|c|}{ Относительные показания к операции при СД } \\
\hline $\begin{array}{l}\text { 1. Выраженный неврологический дефицит } \\
\text { 2. Сепсис }\end{array}$ & 1. Неконтролируемый болевой синдром \\
$\begin{array}{l}\text { 3. Значительное вовлечение в процесс кости с неста- } \\
\text { бильностью позвоночника }\end{array}$ & 2. Отсутствие ответа на консервативную терапию \\
$\begin{array}{l}\text { 4. Существующая или угрожающая деформация по- } \\
\text { звоночника }\end{array}$ & \\
5. Спинальный абсцесс & \\
\hline
\end{tabular}

материалов в инфицированную область может приводить к их колонизации и персистенции инфекции. Осложнения после установки спинальных конструкций могут отсутствовать, если во время санации инфицированной кости и окружающих тканей использовать местное введение антибиотиков. Рекомендуется применять титановые имплантаты, так как они ассоциированы с более низкой частотой рецидивов [5].

Вопрос об оптимальной хирургической тактике при СД остается дискуссионным [3, 5, 25]. Хирургическое лечение СД может проходить в один или два этапа. Если имеется неврологический дефицит, должна проводиться неотложная декомпрессия и стабилизация позвоночника, так как динамика развития неврологических осложнений зависит от быстроты хирургического вмешательства. Двухэтапное вмешательство предпочтительно у пациентов без неврологического дефицита, но имеющих сопутствующую патологию $[5,7,25]$. Второй этап операции проводится спустя одну или две недели после первого [5].

Вид оперативного вмешательства зависит от уровня поражения, степени деструкции и развившихся осложнений. При этом на первом этапе операции производится удаление пораженных и некротизированных тканей посредством корпоротрансверзэктомии, дискэктомии или ламинэктомии. На втором этапе осуществляется восстановление опорной и защитной функций позвоночника [5, 25, 27-29].

По истечении времени иммобилизации пациенту назначается физиолечение, УЗТ, иглорефлексотерапия, ЛФК. Длительное ограничение двигательной активности приводит к атрофии мышц, возвращение к нормальной жизни проводится постепенно с увеличением объемом нагрузки и движений $[1,14,25]$.

\section{Заключение}

Выполненный анализ литературы показал, что ранняя диагностика СД все еще остается сложной задачей, в то же время заболевание требует своевременного адекватного лечения антибактериальными препаратами и контроля за их эффективностью.

Остается также дискутабельным и не в полнее ясным подход к хирургическому методу лечения СД при болевом синдроме (характер и интенсивность боли), что требует проведения дополнительных исследований.

\section{Литература}

1. Gouliouris, T. Spondylodiscitis: update on diagnosis and management / T. Gouliouris, S. H. Aliyu, N. M. Brown // J. Antimicrob. Chemother. -2010 Nov. - Vol. 65, suppl. 3. - P. iii11-24.

2. Спондилодисцит: ранняя диагностика и тактика ведения / И. Ф. Хафизова [и др.] // Казан. мед. журн. - 2016. - Т. 97, № 6. - C. 988-993.

3. Current diagnosis and treatment of spondylodiscitis / R. Sobottke [et al.] // Dtsch. Arztebl. Int. - 2008 Mar. - Vol. 105, N 10. - P. 181-187.

4. Клинические рекомендации по диагностике и лечению воспалительных заболеваний позвоночника и спинного мозга / Ассоциация нейрохирургов России. - М., 2015. $-34 \mathrm{c}$.

5. Treatment of spondylodiscitis / K. Zarghooni [et al.] // Int. Orthop. - 2012 Feb. - Vol. 36, N 2. - P. 405-411.

6. Moraru, I. Neurological point of view Bacterial spondylodiscitis: diagnostic challenges and therapeutic strategies / I. Moraru // Romanian Neurosurgery. - 2012. Vol. 19, N 4. - P. 299-308.

7. Гончаров, М. Ю. Оказание специализированной нейрохирургической помощи пациентам с неспецифическими гнойными заболеваниями позвоночника в условиях крупного промышленного региона / М. Ю. Гончаров, Е. Ю. Левчик // Бюл. ВСНЦ СО РАМН. - 2011. - № 4, ч. 1. - C. $307-310$.

8. Recommendations for diagnosis and treatment of spondylodiscitis / B. L. Fransen [et al.] // Neth. J. Med. 2014 Apr. - Vol. 72, N 3. - P. 135-138.

9. Spondylodiscitis: evaluation of patients in a tertiary hospital 
/ S. Kaya [et al.] // J. Infect. Dev. Ctries. - 2014 Oct. - Vol. 8 , N 10. - P. 1272-1276.

10. Nonspecific pyogenic spondylodiscitis: clinical manifestations, surgical treatment, and outcome in 24 patients / S. Mann [et al.] // Neurosurg. Focus. - 2004 Dec. Vol. 17, N 6. - P. E3.

11. Management of adult spontaneous spondylodiscitis and its rising incidence / A. Sur [et al.] // Ann. R. Coll. Surg. Engl. 2015 Sep. - Vol. 97, N 6. - P. 451-455.

12. Diagnosis and management of adult pyogenic osteomyelitis of the cervical spine / F. L. Jr. Acosta [et al.] // Neurosurg. Focus. - 2004 Dec. - Vol. 17, N 6. - P. E2.

13. Bacteriological features of infectious spondylodiscitis at Mohammed V Military Teaching Hospital of Rabat / A. Zohoun [et al.] // Braz. J. Microbiol. - 2012 Oct-Dec. - Vol. 43, N 4. - P. 1327-1331.

14. Vertebral osteomyelitis, discitis, and spinal epidural abscess in adults [Electronic resource] : guideline summary // Guideline Clearinghouse (NGC) : [Web site]. - 2013 Aug. - Mode of access: https://www.guideline.gov/summaries/ summary/47349/vertebral-osteomyelitis-discitis-and-spinalepidural-abscess-in-adults. - Date of access: 24.01.2018

15. Duarte, R. M. Spinal infection: state of the art and management algorithm / R. M. Duarte, A. R. Vaccaro // Eur. Spine J. - 2013 Dec. - Vol. 22, N 12. - P. 2787-2799.

16. Ардашев, И. П. Вертебральная инфекция / И. П. Ардашев, Е. И. Ардашева // Хирургия позвоночника. - 2009. - № 2. - С. 68-78.

17. Pathogen identification in suspected cases of pyogenic spondylodiscitis / A. F. Sheikh [et al.] // Front. Cell. Infect. Microbiol. - 2017 Mar. - Vol. 7. - P. 60.

18. Comparison of characteristics of culture-negative pyogenic spondylitis and tuberculous spondylitis: a retrospective study / C. J. Kim [et al.] // BMC Infect. Dis. - 2016 Oct. - Vol. 16, N 1. - P. 560.

19. Клиническое руководство по лечению туберкулеза и его лекарственно-устойчивых форм : приказ М-ва здравоохранения Респ. Беларусь, 22 авг. 2012 г., № 939. - Минск,

\section{References}

1. Gouliouris T, Aliyu SH, Brown NM. Spondylodiscitis: update on diagnosis and management. J Antimicrob Chemother. 2010 Nov;65 Suppl 3:iiil1-24. doi: 10.1093/jac/dkq303

2. Khafizova IF, Popova NA, Panyukhov AG, Gumerov FR. Spondylodiscitis: early diagnosis and treatment of. Kazan Med Zhurn. 2016;97(6):988-93. (In Russ.)

3. Sobottke R, Seifert H, Fätkenheuer G, Schmidt M, Gossmann A, Eysel P. Current diagnosis and treatment of spondylodiscitis. Dtsch Arztebl Int. 2008 Mar;105(10):1817. doi: 10.3238/arztebl.2008.0181

4. Assotsiatsiia neirokhirurgov Rossii. Clinical guidelines for the diagnosis and treatment of inflammatory diseases of the spine and spinal cord. Moscow, RF; 2015. 34 p. (In Russ.)

5. Zarghooni K, Röllinghoff M, Sobottke R, Eysel P. Treatment of spondylodiscitis. Int Orthop. 2012 Feb;36(2):405-11. doi: 10.1007/s00264-011-1425-1

6. Moraru I. Neurological point of view Bacterial spondylodiscitis: diagnostic challenges and therapeutic strategies. Romanian Neurosurgery. 2012;19(4):299-308.
2012. $-82 \mathrm{c}$.

20. Diagnostic yield of fluoroscopy-guided biopsy for infectious spondylitis / B. J. Kim [et al.] // Am. J. Neuroradiol. - 2013 Jan. - Vol. 34, N 1. - P. 233-238.

21. Computed tomography-guided percutaneous biopsy for vertebral osteomyelitis: a department's experience / V. Garg [et al.] // Neurosurg. Focus. - 2014 Aug. - Vol. 37, N 2. - P. E10.

22. CT-guided biopsy in suspected spondylodiscitis - the association of paravertebral inflammation with microbial pathogen detection / D. Spira [et al.] // PLoS One. - 2016 Jan. - Vol. 11, N 1. - P. e0146399.

23. CT-guided percutaneous spine biopsy in suspected infection or malignancy: a study of 214 patients / J. Rehm [et al.] // Rofo. - 2016 Dec. - Vol. 188, N 12. - P. 1156-1162.

24. Magnetic resonance imaging findings of early spondylodiscitis: interpretive challenges and atypical findings / J. A. Yeom [et al.] // Korean J. Radiol. - 2016. Vol. 17, N 5. - P. 565-580.

25. Ближайшие результаты лечения гнойных неспецифических заболеваний позвоночника / М. Ю. Гончаров [и др.] // Хирургия позвоночника. - 2005. - № 2. - С. 70-72.

26. IDSA Clinical practice guidelines for the diagnosis and treatment of native vertebral osteomyelitis in adults / E. F. Berbari [et al.] // Clin. Infect. Dis. - 2015 Sep. - Vol. 61, N 6. - P. e26-46.

27. The anterior stand-alone approach (ASAA) during the acute phase of spondylodiscitis: results in 40 consecutively treated patients / G. D'Aliberti [et al.] // Eur. Spine J. - 2012 May. Vol. 21, suppl. 1. - P. S75-82.

28. Posterior fixation without debridement for vertebral body osteomyelitis and discitis / A. S. Mohamed [et al.] // Neurosurg. Focus. - 2014 Aug. - Vol. 37, N 2. - P. E6.

29. Fu, T. S. Minimally invasive percutaneous endoscopic discectomy and drainage for infectious spondylodiscitis / T. S. Fu, L. H. Chen, W. J. Chen // Biomed. J. - 2013 Jul-Aug. - Vol. 36, N 4. - P. 168-173.

Поступила 23.10.2017 2. Принята в печать 31.01.2018 2.

7. Goncharov MYu, Levchik EYu. Provision of specialist neurosurgical care to patients with nonspecific suppurative diseases of the spine in conditions of large industrial region. Biul VSNTs SO RAMN. 2011;(4 ch 1):307-10. (In Russ.)

8. Fransen BL, de Visser E, Lenting A, Rodenburg G, van Zwet AA, Gisolf EH. Recommendations for diagnosis and treatment of spondylodiscitis. Neth J Med. 2014 Apr;72(3):135-8.

9. Kaya S, Ercan S, Kaya S, Aktas U, Kamasak K, Ozalp H, et al. Spondylodiscitis: evaluation of patients in a tertiary hospital. J Infect Dev Ctries. 2014 Oct;8(10):1272-6. doi: $10.3855 /$ jidc. 4522

10. Mann S, Schütze M, Sola S, Piek J. Nonspecific pyogenic spondylodiscitis: clinical manifestations, surgical treatment, and outcome in 24 patients. Neurosurg Focus. 2004 Dec 15;17(6):E3.

11. Sur A, Tsang K, Brown M, Tzerakis N. Management of adult spontaneous spondylodiscitis and its rising incidence. Ann R Coll Surg Engl. 2015 Sep;97(6):451-5. doi: 10.1308/ rcsann.2015.0009

12. Acosta FL Jr, Chin CT, Quiñones-Hinojosa A, Ames CP, 
Weinstein PR, Chou D. Diagnosis and management of adult pyogenic osteomyelitis of the cervical spine. Neurosurg Focus. 2004 Dec 15;17(6):E2.

13. Zohoun A, Akwa EN, Ochi ME, Oragwu N, Akhaddar A, Albouzidi A, et al. Bacteriological features of infectious spondylodiscitis at Mohammed V Military Teaching Hospital of Rabat. Braz J Microbiol. 2012 Oct-Dec;43(4):1327-31. doi: 10.1590/S1517-838220120004000013

14. Vertebral osteomyelitis, discitis, and spinal epidural abscess in adults [Electronic resource]: guideline summary. In: National Guideline Clearinghouse (NGC) [Web site]. 2013 Aug 01. [cited 2018 Jan 24]. Available: https://www.guideline.gov/ summaries/summary/47349/vertebral-osteomyelitis-discitisand-spinal-epidural-abscess-in-adults

15. Duarte R M, Vaccaro AR. Spinal infection: state of the art and management algorithm. Eur Spine J. 2013 Dec;22(12):278799. doi: 10.1007/s00586-013-2850-1

16. Ardashev IP, Ardasheva EI. Vertebral infection. Khirurgiia Pozvonochnika. 2009;(2):68-78. (In Russ.)

17. Sheikh AF, Khosravi AD, Goodarzi H, Nashibi R, Teimouri A, Motamedfar A, et al. Pathogen identification in suspected cases of pyogenic spondylodiscitis. Front Cell Infect Microbiol. 2017 Mar;7:60. doi: 10.3389/fcimb.2017.00060

18. Kim CJ, Kim EJ, Song KH, Choe PG, Park WB, Bang JH, et al. Comparison of characteristics of culture-negative pyogenic spondylitis and tuberculous spondylitis: a retrospective study. BMC Infect Dis. 2016 Oct;16(1):560.

19. Clinical guidelines for the treatment of tuberculosis and its drug-resistant forms: prikaz M-va zdravookhraneniia Resp Belarus' 22 avg 2012 g № 939. Minsk, RB; 2012. 82 p. (In Russ.)

20. Kim BJ, Lee JW, Kim SJ, Lee GY, Kang HS. Diagnostic yield of fluoroscopy-guided biopsy for infectious spondylitis. AJNR Am J Neuroradiol. 2013 Jan;34(1):233-8. doi: 10.3174/ajnr.A3120

21. Garg V, Kosmas C, Young PC, Togaru UK, Robbin MR. Computed tomography-guided percutaneous biopsy for vertebral osteomyelitis: a department's experience. Neurosurg Focus. 2014 Aug;37(2):E10. doi: 10.3171/2014.6.FOCUS14134

22. Spira D, Germann T, Lehner B, Hemmer S, Akbar Ml, Jesser $\mathrm{J}$, et al. CT-guided biopsy in suspected spondylodiscitis - the association of paravertebral inflammation with microbial pathogen detection. PLoS One. 2016 Jan;11(1):e0146399. doi:10.1371/journal.pone.0146399

23. Rehm J, Veith S, Akbar M, Kauczor HU, Weber MA. CTguided percutaneous spine biopsy in suspected infection or malignancy: a study of 214 patients. Rofo. 2016 Dec;188(12):1156-1162. doi: 10.1055/s-0042-116233

24. Yeom JA, Lee IS, Suh HB, Song YS, Song JW. Magnetic resonance imaging findings of early spondylodiscitis: interpretive challenges and atypical findings. Korean J Radiol. 2016 Sep-Oct;17(5):565-80. doi: 10.3348/kjr.2016.17.5.565

25. Goncharov MYu, Sakovich VP, Danilov EP, Cherepanov AV, Volkov IV, Levchik EYu. Early results of treatment of purulent nonspecific diseases of the spine. Khirurgiia Pozvonochnika. 2005;(2):70-2. (In Russ.)

26. Berbari EF, Kanj SS, Kowalski TJ, Darouiche RO, Widmer $\mathrm{AF}$, Schmitt SK, et al. IDSA Clinical practice guidelines for the diagnosis and treatment of native vertebral osteomyelitis in adults. Clin Infect Dis. 2015 Sep;61(6):e26-46. doi: $10.1093 / \mathrm{cid} / \mathrm{civ} 482$

27. D'Aliberti G, Talamonti G, Villa F, Debernardi A. The anterior stand-alone approach (ASAA) during the acute phase of spondylodiscitis: results in 40 consecutively treated patients. Eur Spine J. 2012 May;21 Suppl 1:S75-82. doi: 10.1007/s00586-012-2238-7

28. Mohamed AS, Yoo J, Hart R, Ragel BT, Hiratzka J, Hamilton DK, et al. Posterior fixation without debridement for vertebral body osteomyelitis and discitis. Neurosurg Focus. 2014 Aug;37(2):E6. doi: 10.3171/2014.6.FOCUS14142

29. Fu TS, Chen LH, Chen WJ. Minimally invasive percutaneous endoscopic discectomy and drainage for infectious spondylodiscitis. Biomed J. 2013 Jul-Aug;36(4):168-74. doi: $10.4103 / 2319-4170.112742$

Submitted 23.10.2017

Accepted 31.01.2018

\section{Сведения об авторах:}

Кубраков К.М. - к.м.н., доцент кафедры неврологии и нейрохирургии, Витебский государственный ордена Дружбы народов медицинский университет;

Мигунова В.А. - студентка 6 курса лечебного факультета, Витебский государственный ордена Дружбы народов медицинский университет.

\section{Information about authors:}

Kubrakov K.M. - Candidate of Medical Sciences, associate professor of the Chair of Neurology \& Neurosurgery, Vitebsk State Order of Peoples' Friendship Medical University;

Migunova V.A. - the sixth-year medical student, Vitebsk State Order of Peoples' Friendship Medical University.

Адрес для корреспонденции: Республика Беларусь, 210023, г. Витебск, пр. Фрунзе, 27, Витебский государственный ордена Дружбы народов медицинский университет, ккафедра неврологии и нейрохирургии. Е-таil: k-kubrakov@yandex.ru - Кубраков Константин Михайлович.

Correspondence address: Republic of Belarus, 210023, Vitebsk, 27 Frunze ave., Vitebsk State Order of Peoples'Friendship Medical University, Chair of Neurology \& Neurosurgery.E-mail: k-kubrakov@yandex.ru-Konstantin M. Kubrakov. 